\title{
PAROXYSMAL TACHYCARDIA IN PREGNANCY
}

\author{
BY \\ PAUL SZEKELY AND LINTON SNAITH
}

From the Cardiovascular Department and the Department of Obstetrics, Newcastle General Hospital

Received November 7, 1952

There are conflicting views regarding the incidence of paroxysmal tachycardia in pregnancy. Jensen (1938) concluded from a review of the literature that there had been only 19 cases reported up to 1938 in which the diagnosis was beyond reasonable doubt, and he thought that the condition was rare during pregnancy. On the other hand, Campbell (1947) was of the opinion that paroxysmal tachycardia is far more common in pregnancy than is generally recognized. The purpose of this paper is to review briefly the relevant papers and to analyse ten personally observed cases.

Campbell and Elliott (1939) discussed the influence of pregnancy in 7 of a series of 100 patients with paroxysmal tachycardia. - Jensen et al. (1940) observed three cases of paroxysmal tachycardia in a series of 108 pregnant patients with organic heart disease. Hamilton and Thomson (1941) stated that they were able to make a satisfactory diagnosis of paroxysmal tachycardia during pregnancy in 13 out of 781 patients with rheumatic heart disease. Browning and Clark (1941) reported two cases of paroxysmal tachycardia occurring during pregnancy. Single cases were reported by Weyler and Dustin (1942), Lian et al. (1948), and by Abbas and Guild (1951). Vander Veer and Kuo (1950) studied 409 pregnant patients with heart disease and found paroxysmal tachycardia in six. They considered this to be a high incidence and suggested that pregnancy may predispose to paroxysmal tachycardia.

\section{Personal ObSERVATIONS}

We were able to make a satisfactory diagnosis of paroxysmal tachycardia in pregnancy in ten cases. There were several more patients who complained of attacks of palpitation, with sudden onset and offset, and who very probably were suffering from true paroxysmal tachycardia, but in the absence of electrocardiographic or clinical confirmation were not included in this series. The ten cases analysed in the present paper were observed during a ten-year period (1942-52). The total number of patients with heart disease seen and followed up in one or more pregnancies during this ten-year period was 380 . The total number of pregnant patients delivered in the department of obstetrics during the same period was 10,746 .

Six patients had rheumatic heart disease, and in four no lesions were detected.

There were four primiparæ and six multiparæ. Two of the multiparæ had attacks in more than one pregnancy. One patient had attacks in three successive pregnancies but never between pregnancies.

Paroxysms of tachycardia occurred during pregnancy in eight patients. In six of these the attacks appeared in late pregnancy, and in two they started early and became more frequent in the latter months of pregnancy. One patient who had frequent attacks during pregnancy developed an attack during labour. Attacks occurred during the puerperium in six patients. Two of these had attacks in the puerperium only and none during pregnancy or labour. 
In eight patients we were able to obtain cardiograms during paroxysms of tachycardia. In all eight the tachycardia was of the supraventricular type (Fig. 1).

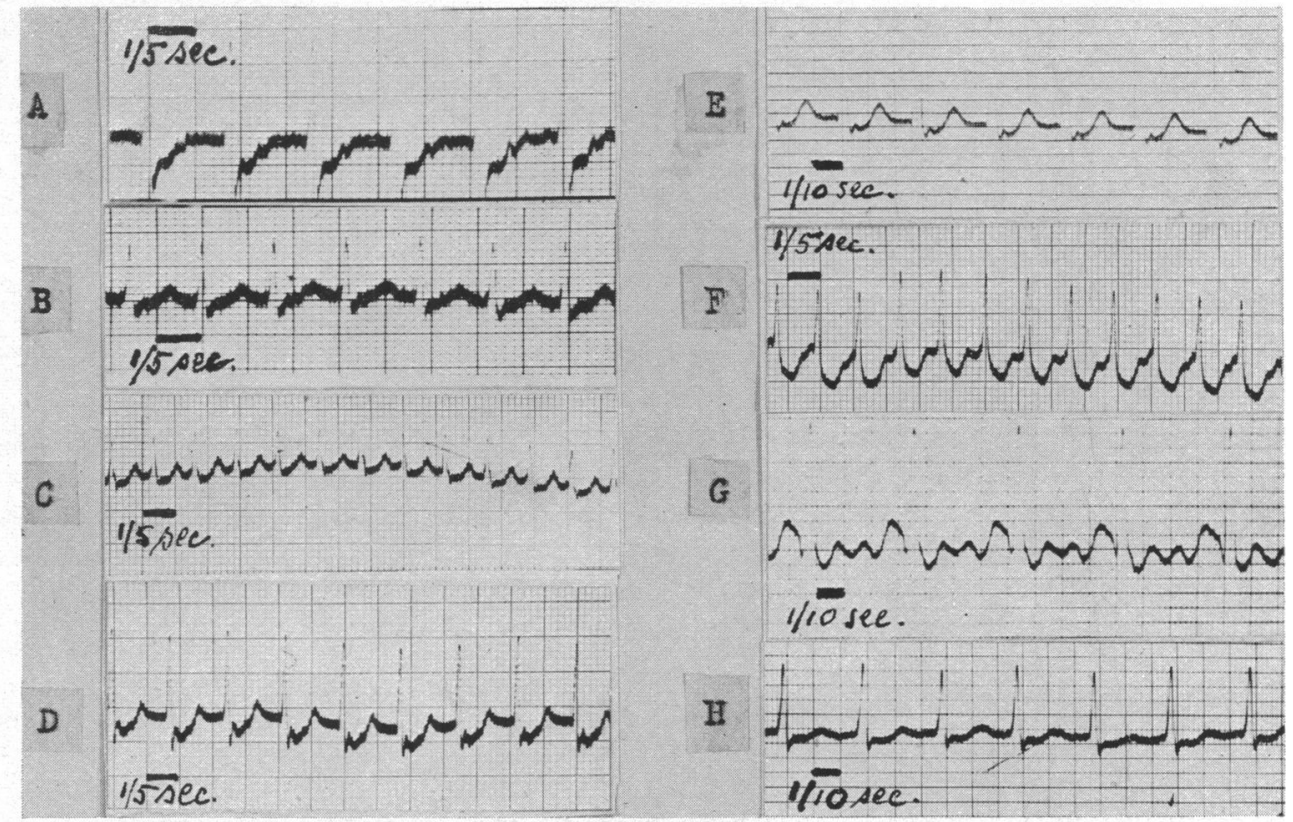

Fig. 1.-Lead II in eight cases (A-H) of paroxysmal supraventricular tachycardia complicating pregnancy.

\section{Discussion}

The observations of Anderson $(1932,1933)$ in two patients with paroxysmal tachycardia are of interest: one who had frequent attacks since the age of six years was free from attacks during her pregnancy and had only one attack seven days after delivery; the other had attacks only during pregnancies and never between pregnancies. Similarly, Campbell and Elliott (1939) said that in two of their patients the attacks became less frequent during pregnancy, while five patients were unfavourably influenced by pregnancy. In 19 cases details are available as to the appearance of the first paroxysm: eleven patients had attacks prior to pregnancy while in eight the attacks first appeared in pregnancy or in the puerperium. In our series, in one case the history was inadequate because of the mental condition of the patient; in the remaining nine the first attack occurred during pregnancy or in the puerperium. Six out of these nine patients had paroxysms only in relation to pregnancy while three had occasional attacks during a followup period of 32 months, 28 months, and 3 months, respectively, following delivery. Accordingly, these observations favour the idea that pregnancy tends to increase susceptibility to paroxysmal tachycardia. Four of the patients in the present series were receiving digitalis before the onset of paroxysmal tachycardia, but it is very unlikely that digitalis caused the attacks in any of these four.

In nearly all cases, including the present group, the tachycardia was of the supraventricular type. As far as we are aware the only published case of ventricular tachycardia confirmed by electrocardiogram occurring during pregnancy is that of McMillan and Bellett (1931-32); in this case frequent paroxysms occurred in the latter part of pregnancy and the patient was delivered by cæsarian section during such a paroxysm. Mackenzie (1921) mentions a patient who was studied with the polygraph and who was thought to have paroxysmal ventricular tachycardia during pregnancy. The electrocardiogram in one of our cases shows a $2: 1 \mathrm{~A}-\mathrm{V}$ block (Fig. 1G). This 
tracing may be regarded by some as an example of auricular flutter, but the various clinical and cardiographic features observed in this patient conform to the definition of paroxysmal tachycardia as postulated by Evans (1944). Evans (1944) and also Campbell (1945) have drawn attention to the occurrence of A-V block in paroxysmal tachycardia. It is possible that the tracings of some of our other patients also showed 2:1 A-V block, but adequate chest leads were not available in each instance for the study of this special point. Two of our patients also had auricular fibrillation, and this interchangeability of rhythm has also been observed (Evans, 1944).

According to Carr and Hamilton (1933) paroxysmal tachycardia does not make pregnancy dangerous provided there is no structural heart disease; on the other hand, it can be a dangerous complication if mitral stenosis is present. They observed heart failure in several patients, with a fatal outcome in one, among their seven cases of heart disease complicated by paroxysmal tachycardia. Campbell (1947a) and Gilchrist and Haig (1950) have also stated that the prognosis entirely depends on the underlying disease. Our observations show that paroxysmal tachycardia in the presence of organic heart disease can precipitate heart failure: it did so on four occasions in three patients. The attacks that were associated with heart failure lasted 12 hours, 6 hours, 72 hours, and 24 hours, respectively. In three patients with rheumatic heart disease the paroxysms were at no time associated with heart failure. Here, the longest attack lasted 1 hour, 1 hour, and $1 \frac{1}{2}$ hours, respectively. Similarly, no heart failure developed in four patients without structural heart disease. In these four patients the longest attack lasted 17 hours, 1 hour, 46 hours, and $\frac{1}{2}$ hour, respectively. From these observations it would appear that a long paroxysm of tachycardia in the presence of organic heart disease must be considered as a serious complication and every effort should be made to restore normal rhythm as soon as possible. It is also our impression that paroxysmal tachycardia is of more serious consequence during pregnancy than in the puerperium, but the numbers are not large enough to enable us to draw definite conclusions in this respect.

Hamilton and Thomson (1941) used ipecacuanha with success in five patients with paroxysmal tachycardia in the puerperium and in one patient during pregnancy. They observed no ill effects and suggested that the drug can be safely used during pregnancy and the puerperium in the absence of congestive heart failure or recent operation when violent vomiting is to be avoided. They also had good results with digitalis; occasionally, they used quinidine. Browning and Clark (1941) and Abbas and Guild (1951) also used quinidine. These authors believe that there is no adequate evidence to support the general belief that quinidine or associated alkaloids precipitate labour. According to Sollmann (1942) quinine or quinidine has no effect on the uterus unless labour pains have started. We used digitalis in six patients-oral medication in four, and intravenous followed by oral medication in two. The impression gained was that in four patients digitalis was responsible for the termination of the attack, while in two patients spontaneous termination was a strong probability. One patient was given procaine amide, after termination of the attack, $2 \frac{1}{2}$ grams daily for 4 weeks, in an attempt to prevent recurrences. However, she had a recurrence during procaine amide medication on the eighth day following mitral valvotomy. It is possible that the dose was inadequate. Four patients received no drugs; in one of these the attack stopped after violent vomiting. We have not used quinidine during pregnancy so far, but procaine amide which has a quinidine-like action on the heart was used for one patient without any harmful effect on the course of pregnancy. Pressure on the carotid sinus failed to restore normal rhythm in six patients.

\section{SUMMARY}

Ten cases of paroxysmal tachycardia complicating pregnancy are reported. Paroxysms of tachycardia occurred during pregnancy in eight patients, during labour in one, and during the puerperium in six. The present observations favour the idea that pregnancy tends to increase susceptibility to paroxysmal tachycardia.

Paroxysmal tachycardia in the absence of structural heart disease does not seriously interfere with the normal course of pregnancy. On the other hand, if associated with structural heart 
disease it can precipitate heart failure. This statement is based on observations on rheumatic heart disease; no cases of paroxysmal tachycardia associated with cardiac disease other than rheumatic heart disease were seen during pregnancy.

We are grateful to Dr. W. G. A. Swan for his criticism and help. We also wish to thank Dr. R. Orton for permission to include a case, under his care.

\section{REFERENCES}

Abbas, T. M., and Guild, A. A. (1951). J. Obstet. Gynaec. Brit. Emp., 58, 437.

Anderson, D. F. (1932). Brit. med. J., 1, 657.

- (1933). Brit. med. J., 1, 224.

Browning, J. S., and Clark, C. J. (1941). J. Indiana State med. Ass., 34, 21.

Campbell, M. (1945). Brit. Heart J., 7, 183.

(1947). Guy's Hosp. Gaz., 61, 169.

(1947a). Lancet, $2,641$.

二, and Elliott, G. H. (1939). Brit. Heart J., 1, 123.

Carr, F. B., and Hamilton, B.E. (1933). Amer. J. Obstet. Gynaec., 26, 824.

Evans, W. (1944). Brit. Heart J., 6, 221.

Gilchrist, A. R., and Haig, D. C.(1950). Heart Disease and Pregnancy in Modern Trends in Obstetrics and Gynrecology, edited by K. Bowes. London: Butterworth and Co.

Hamilton, B. E., and Thomson, K. J. (1941). The Heart in Pregnancy and the Childbearing Age. Boston: Little, Brown and Co.

Jensen, J. (1938). The Heart in Pregnancy. St. Louis: C. V. Mosby Co.

-, Wegner, C., Keys, E. H., and Smith, H. R. (1940). Amer. J. Obstet. Gynac., $39,443$.

Lian, C., Golblin, V., and Benzecry, J. (1948). Paris med., 38, 272.

Mackenzie, J. (1921). Heat Disease and Pregnancy. Oxford Medical Publication.

McMillan, T. M., and Bellett, S. (1931-32). Amer. Heart J., 7, 70.

Sollmann, T. (1942). A Manual of Pharmacology. 6th ed., Philadelphia: W. B. Saunders and Co.

Vander Veer, J. B., and Kuo, P. T. (1950). Amer. Heart J., 39, 2.

Weyler, H., and Dustin, C. C. (1942). New Engl. J. Med., 227, 785. 